AperTO - Archivio Istituzionale Open Access dell'Università di Torino

\title{
Platelet-derived Growth Factors and Receptors in Canine Lymphoma
}

\section{This is the author's manuscript}

Original Citation:

Availability:

This version is available http://hdl.handle.net/2318/155453

since 2016-09-21T16:48:41Z

Published version:

DOI:10.1016/j.jcpa.2014.07.001

Terms of use:

Open Access

Anyone can freely access the full text of works made available as "Open Access". Works made available under a Creative Commons license can be used according to the terms and conditions of said license. Use of all other works requires consent of the right holder (author or publisher) if not exempted from copyright protection by the applicable law. 


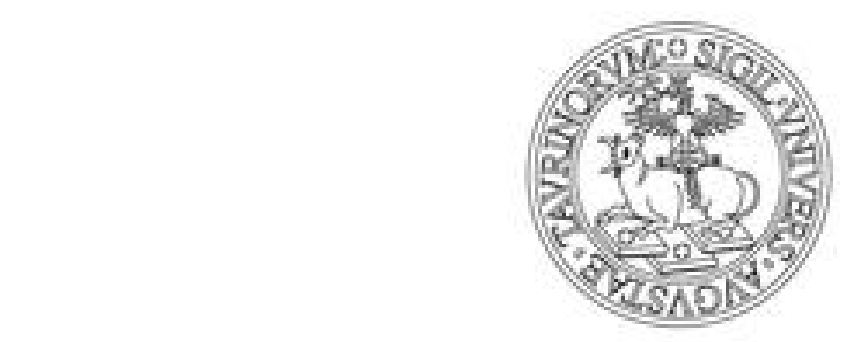

\section{UNIVERSITÀ DEGLI STUDI DI TORINO}

This Accepted Author Manuscript (AAM) is copyrighted and published by Elsevier. It is posted here by agreement between Elsevier and the University of Turin. Changes resulting from the publishing process - such as editing, corrections, structural formatting, and other quality control mechanisms - may not be reflected in this version of the text. The definitive version of the text was subsequently published in JOURNAL OF COMPARATIVE PATHOLOGY, , 2014, 10.1016/j.jcpa.2014.07.001.

You may download, copy and otherwise use the AAM for non-commercial purposes provided that your license is limited by the following restrictions:

(1) You may use this AAM for non-commercial purposes only under the terms of the CC-BY-NC-ND license.

(2) The integrity of the work and identification of the author, copyright owner, and publisher must be preserved in any copy.

(3) You must attribute this AAM in the following format: Creative Commons BY-NC-ND license (http://creativecommons.org/licenses/by-nc-nd/4.0/deed.en), 10.1016/j.jcpa.2014.07.001

The definitive version is available at: http://linkinghub.elsevier.com/retrieve/pii/S0021997514001157 


\section{PDGF and its receptors in canine lymphoma}

A. Aricò*, E. Guadagnin*, S. Ferraresso*, M.E. Gelain*, S. Iussich ${ }_{\dagger}$, B.C. Rütgen ${ }^{+}$, S. Mazzariol*, L. Marconato ${ }^{*}$, L. Aresu*

* Department of Comparative Biomedicine and Food Science, University of Padova, Viale dell'Università 16, 35020 Agripolis Legnaro PD, Italy

† Department of Veterinary Science, University of Turin, Via Leonardo da Vinci 44, 10095 Grugliasco TO, Italy

${ }^{+}$Department of Pathobiology, University of Veterinary Medicine of Vienna, Veterinärplatz

1, 1210, Vienna, Austria

*entro Oncologico Veterinario, via San Lorenzo 1-4, 40037 Sasso Marconi BO, Italy

Correspondence to: L. Aresu (e-mail address: luca.aresu@unipd.it)

\section{Summary}

Lymphoma is the most common haematopoietic tumour in dog. Commonly, dogs develop aggressive multicentric lymphoma, showing overlapping features with human non-Hodgkin lymphoma (hNHL). Several studies have demonstrated that Platelet-Derived Growth Factors (PDGF) and its receptors play an important role in the pathogenesis of hNHL, in particular of T-cell lymphoma. In canine lymphoma the role of these molecules is unknown. The aim of the study was to investigate the gene and protein expression of PDGF-A, PDGF-B, PDGFR- $\alpha$ and PDGFR- $\beta$ in canine aggressive B-cell and T-cell lymphomas. Forty-one dogs were enrolled in this study: 19 dogs were affected by Diffuse Large B-cell Lymphoma (DLBCL), 5 by Peripheral T-cell Lymphoma (PTCL) and by 3 T-Lymphoblastic Lymphoma (T-LBL), 9 with reactive hyperplasia and 5 as healthy controls. CLBL-1 and OSW cell lines were also used in this study. In PTCLs and T-LBLs both PDGF-B transcript and protein levels were significantly higher respect to B-cell counterpart; indeed, PDGF-B was not expressed by T-cells in non-neoplastic lymph nodes. PDGFR- $\alpha$ and PDGFR- $\beta$ in control lymph nodes were minimally expressed by lymphocytes and plasma cells, mostly in the follicles, whereas neoplastic T-cells showed similar positive immunostaining for both 
receptors. Comparing the gene expressions, significant correlations were found in DLBCL between PDGFR- $\beta$ and both PDGF-A and PDGF-B; whereas considering the protein expressions, PDGFR-B and both PDGFRs were significantly correlated in PTCLs and T-LBLs. We conclude that canine Tcell lymphoma might represent a new and important source of PDGF-B and PDGFRs and that the expression of these molecules may suggest a functional autocrine and/or paracrine loop of growth stimulation, which causes different cellular effects and responses.

Keywords: angiogenic factors, canine lymphoma, PDGF, therapy.

\section{Introduction}

Lymphoma, the most common haematopoietic tumour in dogs, is a generic term to describe a heterogeneous disease entity, encompassing many subtypes of varying morphology, pathophysiology and clinical features (Ponce et al., 2010; Valli et al., 2011; Aresu et al., 2013). Commonly, dogs develop aggressive multicentric lymphoma, showing overlapping features with human non-Hodgkin lymphoma (hNHL) (Marconato et al., 2013). Emerging data on the proangiogenic properties of lymphoma cells and the mechanisms of vascular assembly suggest that angiogenesis is highly relevant to the biology and therapy of hNHL (Moehler et al., 2003; Ruan et al., 2013). Several studies have demonstrated that cytokines involved in angiogenesis play an important role in the pathogenesis of hNHL, contributing to its progression in an autocrine and paracrine fashion on lymphocytes (Pedersen et al., 2005; Labidi et al., 2010). In this contest Platelet-Derived Growth Factors (PDGFs) are a pleotrophic family of peptide growth factors that signal through cell surface tyrosine kinase receptors (TKRs), and stimulate various cellular functions including growth, proliferation, and differentiation (Heldin and Westermark, 1999). To date, the PDGF pathway has been described in a wide range spectrum of human tumours, including T-cell lymphomas (Su and Kadin, 1989; Piccaluga et al., 2005). The biologic role of PDGF 
signalling can vary from autocrine stimulation of cancer cell growth to subtler paracrine interactions involving adjacent stroma and vasculature. Experimental studies have shown that the concomitant expression of PDGF ligands and receptors by the same neoplastic cell contributes to cancer progression by creating an autocrine loop (Heldin and Westermark, 1999). PDGF may also modulate the expression of other angiogenic factors, such as Vascular Endothelial Growth Factor (VEGF) whose receptors (Flt-1, KDR/Flk-1 and Flt-4) are also classified as RTKs (Halper, 2010). Recently, we suggested a potential role of VEGF in the pathogenesis of canine lymphoma (Aresu $e t$ al., 2012; Aricò et al., 2013). The role of PDGF and its receptors in canine lymphoma is still unknown. The aim of the study was to investigate the gene and protein expression of PDGF-A, PDGF-B, PDGFR- $\alpha$ and PDGFR- $\beta$ in canine B-cell and T-cell lymphomas. Two canine lymphoma cell lines were also tested to elucidate the possible involvement of PDGF and PDGFRs without the confounding influence of the tumour microenvironment.

\section{Materials and Methods}

\section{Case Selection and Tissue Sampling}

For this study, 36 dogs were enrolled after complete staging work-up, including physical examination, complete blood cell count, peripheral blood and bone marrow aspirate, thoracic radiography, abdominal ultrasound and lymphadenectomy for histopathological and immunohistochemical diagnosis according to WHO (Valli et al., 2011). Five control lymph nodes were obtained from pathogen-free adult dogs. For the purposes of the study, tumour samples were immediately divided into aliquots and stored for different analytical techniques. For histological examination and immunohistochemistry, the tissue was formalin-fixed and paraffin embedded. For RNA isolation, aliquots of approximately $100 \mathrm{mg}$ of neoplastic tissue were immersed in RNAlater® solution (Applied Biosystems, Foster City, CA) and stored at $-20^{\circ} \mathrm{C}$ until use. Owners of dogs with lymphoma were offered to treat their animals with multidrug chemotherapy, consisting of 
doxorubicin, vincristine, cyclophosphamide, L-asparaginase and prednisone. For selected dogs, lymph node excision was also obtained at the end of the chemotherapeutic protocol and/or at relapse. The study was approved by the University Committee (protocol 20085MSFH2); a written consent was obtained from all owners.

\section{Cell lines}

Canine cell lines used in this study included: B-cell lymphoma cell line CLBL-1 (Rütgen et al., 2010) and T-cell lymphoma cell line OSW (Kisseberth et al., 2007). The cell culture conditions were described previously by Rütgen et al. (2010).

\section{Quantitative real-time RT-PCR ( $q R T$ - $P C R)$}

Total RNA was isolated from tissue samples using TRIzol@ (Invitrogen, Carlsbad, CA) according to the manufacturer's instruction. Total RNA concentration and quality were measured with a Nanodrop ND-1000 spectrophotometer (Nanodrop Technologies, Wilmington, DE). First-strand cDNA was synthesised from $300 \mathrm{ng}$ of total RNA using Superscript II (Invitrogen, Life Technologies, Carlsbad, CA) according to the manufacturer's protocol. The generated cDNA was used as the template for quantitative real-time RT-PCR (qRT-PCR) in a LightCycler 480 Instrument (Roche Diagnostics, Basel, Switzerland) using standard PCR conditions. The qRT-PCR reactions consisted of $5 \mu \mathrm{l}$ of Platinum SYBR Green qPCR SuperMix-UDG (Invitrogen, Life Technologies, Carlsbad, CA), $0.3 \mu \mathrm{l}$ of forward and reverse primers $(10 \mu \mathrm{M})$ (the primer combination and final concentrations were optimized during assay setup) and $2.5 \mu \mathrm{l}$ of diluted ( 1 to 100$) \mathrm{cDNA}$. The primers, shown in Table 1, were designed using Primer Express 2.0 (Applied Biosystem, Life Technologies, Carlsbad, CA). Calibration curves using a 7-fold serial dilution (1:2) of a cDNA pool revealed PCR efficiencies near 2.0 and error values $<0.2$. Canine transmembrane BAX inhibitor 
motif containing 4 (CGI-119) was chosen as reference gene for the absence of pathological state dependent differences in mRNA expression, as reported by Aricò et al. (2013). $\Delta \Delta \mathrm{Ct}$ method (Livak and Schmittgen, 2001) was used for the relative quantification of mRNA, ultimately expressed as Relative Quantification (RQ). Cell lines reactions have been made in duplicate.

\section{Histological and immunohistochemical examination}

For histological examination, 3- $\mu \mathrm{m}$ section slides were stained with haematoxylin and eosin. For lymphoma phenotyping, an immunohistochemical panel of selected antibodies was applied, including: a monoclonal Mouse Anti-Human CD3 (Clone F7.2.38, Dako, Atlanta, GA, USA, T cells; diluted 1 in 100), a monoclonal Mouse Anti-Human CD5 (Clone CD5/54/F6, Dako, T cells; diluted 1 in 100), a monoclonal Mouse Anti-Human CD79acy (Clone HM57, Dako, all stages of Bcells; diluted 1 in 100) and a CD20 Epitope Specific Rabbit Antibody (RB-9013-P, Thermo Fisher Scientific Inc, Cheshire WA7 1TA, UK, mature B cells; diluted 1 in 800). The primary antibody incubation step was performed by an automated system for all antibodies (Ventana Medical Systems). Immunohistochemical analysis for PDGF and receptors was performed using 3\% hydrogen peroxide in methanol for $30 \mathrm{~min}$ at room temperature for blocking endogenous peroxidase activity. Sections underwent high-temperature antigen unmasking by incubation with $98^{\circ} \mathrm{C}$ citric acid buffer ( $\mathrm{pH}$ 6). The antibodies used in this study were the following: a mouse monoclonal PDGFA (N-30: sc-128, Santa Cruz Biotechnology Inc., Santa Cruz, California, USA; dilution 1:400), a rabbit Polyclonal PDGFB (H-55: sc-7878, Santa Cruz Biotechnology; dilution 1:400), a rabbit Polyclonal PDGFR- $\alpha$ (C-20: sc-338, Santa Cruz Biotechnology; dilution 1:100) and a rabbit Polyclonal PDGFR- $\beta$ (958: sc-432, Santa Cruz Biotechnology; dilution 1:200) (Maniscalco et al., 2013). Antibodies were detected using an avidin-biotin peroxidase complex technique with the Vectastain Elite ABC Kit (Vector Laboratories Inc., Burlingame, CA, USA). For negative controls, the sections were incubated without the primary antibodies. Cytoplasmic immunolabelling of the 
antibodies was evaluated in neoplastic cells in lymphomas and in the normal lymphocytes in the control lymph nodes. Quantification of protein expression was assessed in 10 randomly selected fields with a 40x objective. The intensity and the percentage of labelled tumour cells were recorded. An intensity score of 0 was given if no staining was detected, 1 if there was weak to moderate staining, 2 if moderate to strong staining was present, and 3 if strong staining was detected. A total score for each examined field was obtained by multiplying the intensity score by the percentage of immunoassayed cells. Fibroblasts, vascular smooth muscle cells and endothelial cells, within and surrounding the tumour and in the normal lymph nodes were used as internal positive controls and excluded from the evaluation. An image analysis system that consisted of an Olympus BX51 microscope and software analysis (analySIS, Soft imaging system, Münster, Germany) was used. All evaluations were performed in blinded fashion by three independent pathologists (LA, AA and SI).

\section{Statistical analysis}

Grubbs' test was used to identify potential outliers. The statistical analysis of gene and protein expression data was performed using the non-parametric Kruskal-Wallis test followed by Dunn's post-test. A non-parametric Spearman correlation analysis was used to determine relationships among target genes and coded proteins. A non-parametric paired t test data (Wilcoxon signed rank test) was used to identify differences in gene and protein expression between samples. GraphPad Prism 5 software (San Diego, California, USA) was used for all statistical evaluations. Statistical significance was set at $p<0.05$.

\section{Results}


Forty-one dogs were enrolled in this study: 27 with lymphoma, 9 with reactive hyperplasia and 5 as healthy controls. At the time of admission 19 dogs were affected by Diffuse Large B-cell Lymphoma (DLBCL), 5 by Peripheral T-cell Lymphoma (PTCL) and by 3 T-Lymphoblastic Lymphoma (T-LBL). At the end of treatment, 2 dogs affected by DLBCL and 2 dogs affected by PTCL were in relapse, whereas 8 dogs were in remission status from DLBCL, with diagnosis compatible with reactive hyperplasia. Among the 8 dogs that were in remission status from DLBCL at the end of the therapy, two experienced a relapse during the course of the study. Among the remainder of 27 dogs with lymphoma, 8 DLBCLs, 3 T-LBLs and 3 PTCLs died before the end of the treatment because of lymphoma-related causes. One DLBCL has not completed the therapy yet.

$q R T-P C R$

qRT-PCR assay results are summarized in Table 1 Supplementary Material. PDGF-A, PDGFR- $\alpha$ and PDGFR- $\beta$ mRNA levels in control lymph nodes were significantly higher compared to DLBCLs, PTCLs and T-LBLs $(p<0.05)$. A significantly higher PDGF-B mRNA expression was observed in PTCLs and T-LBLs compared to DLBCLs $(p<0.05)$. On the other hand, PDGF-B transcript amount was lower in the DLBCLs compared to the control lymph nodes (Fig. 1). mRNA levels of all genes in cell lines were not quantifiable or very low, with the exception of PDGF-A and PDGFR- $\alpha$ mRNA expression in OSW. In dogs with DLBCL at diagnosis significant correlations were found between PDGFR- $\beta$ and both PDGF isoforms (PDGF-A versus PDGFR- $\beta$ $\mathrm{r}=0.58, p<0.0041$; PDGF-B versus PDGFR- $\beta \mathrm{r}=0.63, p<0.0014)$, whereas in the 8 dogs in remission status from DLBCL at the end of therapy, a significant correlation was found between PDGF-A and PDGFR- $\alpha(\mathrm{r}=0.76, p<0.036)$. In the same dogs a significant expression trend $(\mathrm{p}<0.05)$ was also found, with the paired t-test analysis, at the time of admission and at the end of therapy for both PDGF isoforms and their receptors, with higher mRNA levels at the end of treatment. In addition, in the two dogs that experienced a relapse, mRNA levels of all genes were higher but not significantly at the end of chemotherapy respect to diagnosis and to relapse (data not shown). 


\section{Immunohistochemical analysis}

Immunohistochemical results are summarized in Table 2 Supplementary Material and showed in Fig. 2. In non-neoplastic lymph nodes, PDGF-A and PDGF-B were moderately expressed in the follicles and not expressed in the paracortex (Fig. 3a, 3d). Moderate expression of both PDGFRs was found in lymphocytes located in areas ascribable to marginal zone and germinal center. Focally, lymphocytes in the paracortex and medulla expressed both receptors (Fig. 3g, 31). PDGFR$\alpha$ and PDGFR- $\beta$ protein expression was significantly higher in the DLBCLs compared to control lymph nodes (Fig. 3h, 3j). Interestingly, in the PTCLs and T-LBLs, PDGF-B was significantly higher compared to control lymph nodes and DLBCLs (Fig. 3f), whereas PDGFR- $\alpha$ was significantly higher only compared to control lymph nodes (Fig. 3i). In dogs with PTCLs and TLBLs significant correlations were found between PDGFR-B and both PDGFRs (PDGF-B versus PDGFR- $\beta \mathrm{r}=0.70, p<0.005$; PDGF-B versus PDGFR- $\alpha \mathrm{r}=0.79, p<0.002)$. Interestingly, the paired $\mathrm{t}$ test analysis in the 8 dogs that were in remission status from DLBCL at the end of therapy, showed that protein levels of PDGF-A, PDGF-B, PDGFR- $\alpha$ and PDGFR- $\beta$ decreased at the end of treatment; in particular the two receptors had a significantly higher expression at diagnosis compared to the results at the end of therapy. All markers were strongly detected in OSW, whereas CLBL-1 presented only a weak cytoplasmatic immunostaining.

\section{Discussion}

The involvement of PDGF and its receptors have been described in several human hematopoietic malignancies (Ho et al., 2005; Renné et al., 2005; Karabatsou et al., 2005; Karabatsou et al., 2006; Ria et al., 2008; Piccaluga et al., 2014). The existence of an autocrine and/or paracrine loop contributing to the uncontrollable growth of malignant cells has been also correlated to the clinical outcome in PTCL (Piccaluga et al., 2005, Piccaluga et al., 2014). The role of these molecules has 
never been investigated in canine lymphoma. The aim of this study was to investigate the gene and protein expression of PDGF-A, PDGF-B, PDGFR- $\alpha$ and PDGFR- $\beta$ in a group of dogs with DLBCL, PTCL and T-LBL. Two canine lymphoma cell lines were also used to elucidate the possible involvement of these molecules without the confounding influence of microenvironment in the pathogenesis of this tumour.

In DLBCLs, PDGFs and receptors mRNA were significantly lower when compared to control lymph nodes. However, these results were discordant with the protein assays by immunohistochemistry where PDGFR- $\alpha$ and PDGFR- $\beta$ protein expression was significantly higher in DLBCLs compared to the control lymph nodes. Regarding PTCLs and T-LBLs, PDGF-A, PDGFR- $\alpha$ and PDGFR- $\beta$ mRNA levels were significantly lower compared to the control lymph nodes. The protein levels of the two receptors showed a higher expression in both histotypes compared with controls.

A possible explanation for the discordance between mRNA and protein expression in lymphomas and controls may be related to the presence of a variable number of cells in non neoplastic lymph nodes and in the perinodal tissue, such as fibroblasts, plasma cells, vascular smooth muscle cells and endothelial cells that constitutively express PDGFs and its receptors (Alvarez et al., 2006). Thus, in the control lymph nodes, stroma showed the most considerable protein expression (not scored) and only lymphocytes located in the follicles showed a weak immunostaining. Whereas, in lymphoma-affected tissues the immunolabelling scores were referred to the neoplastic population; moreover in this study we selected histotypes characterized by a diffuse growth in the nodes.

Interestingly, both PTCLs and T-LBLs showed significantly higher PDGF-B transcript and protein amount compared with DLBCLs. PDGFR- $\alpha$ and PDGFR- $\beta$ were minimally expressed by lymphocytes and plasma cells in control lymph nodes, mostly in the follicles, whereas neoplastic Tcells showed high positive immunostaining for both receptors. PDGFRs mRNA is consistently overexpressed in people with PTCL, in particular Piccaluga and colleagues (2014) demonstrated that PDGFR- $\alpha$ was overexpressed in PCTL compared with normal T-cells. Preliminary in vitro and 
ex vivo analysis suggested that PDGFRs are sensitive to inhibition by imatinib and emerging data on the pro-angiogenic properties of lymphoma cells and the mechanisms of vascular assembly reveal that angiogenesis is highly relevant to human T-cell lymphoma (Moehler et al., 2003). In this contest, PDGF is also able to modulate the expression of different stromal angiogenic factors, including VEGF (Ferrara et al., 2003). In previous works we demonstrated that VEGF is correlated with the stage of the disease and the grade in T-cell lymphomas (Aresu et al., 2012; Aricò et al., 2013). In vivo angiogenesis models are still not able to determine whether PDGF-B induces neovascularization via direct action upon endothelial cells, or indirectly recruiting other cells, but the identification of these two targets in the angiogenic process of canine T-cell lymphoma may suggest that PDGF, together with VEGF, might contribute to the clinical aggressive behaviour of Tcell lymphomas. Therefore, it is plausible that the association of PDGFR antagonists and VEGF inhibitors may have a promising future in the therapeutic setting.

Comparing the gene expression results, significant correlations were found between PDGFR- $\beta$ and both PDGF-A and PDGF-B in DLBCLs at time of diagnosis, whereas a significant correlation was only found between PDGFR- $\alpha$ and PDGF-A in dogs that were in DLBCL remission status at the end of therapy. Furthermore, comparing the protein expressions, PDGF-B and both PDGFRs were significantly correlated in T-cell lymphomas. The two PDGF receptor types mediate similar cellular responses. After activation, both receptors stimulate cell proliferation and rearrangement of actin filaments, while only PDGFR- $\beta$ mediates a potent chemotactic response (Heldin and Westermark, 1999). Thus, it is possible that different combinations of PDGF isoforms and receptors lead to cellular effects and responses that are distinctive hallmarks of the disease.

Interestingly, dogs that were in remission status from DLBCL at the end of therapy presented significantly higher gene expression at the end of treatment respect to transcript amount at diagnosis for all molecules. This trend was opposite to protein results at the time of admission and in remission, with significance only for PDGFR- $\alpha$ and PDGFR- $\beta$. Similar PDGF and PDGFRs expression profiling seen in the control lymph nodes and the case of reactive hyperplasia, could 
explain the biological behaviour of the stromal reactive cells, which may effectively contribute to the expression of these molecules (Piccaluga et al., 2014). Considering the findings obtained in cell lines, PDGF-A and PDGFR- $\alpha$ mRNA expression in OSW was the only highlighted. PDGF-A and PDGFR- $\alpha$ appeared to be constitutively released in this cell line and without the influence of microenvironment. Even though it may be a limitation the use of only one cell line.

In conclusion, PDGF-B and PDGFRs seem to be mainly involved in the pathogenesis of PTCLs and T-LBLs and the different expression of PDGFs and PDGFRs at diagnosis and during remission may suggest a functional autocrine and/or paracrine loop of growth stimulation. However, further investigations are needed to assess the ligand-induced activation of these receptors and PDGF signalling in dogs. Even if an autocrine receptor activation may occur in T-cell lymphomas, it is still not known if this is a critical or a contributing event in its development. Also, structural aberrations of PDGFRs that lead to overexpression or expression of abnormal proteins have been described in canine vascular tumours (Abou Asa et al., 2013), and will be taken into consideration in the next studies. PDGF-B and PDGFRs may represent new therapeutic target in canine T-cell lymphomas and future studies will be directed to collect clinical data to further understand a potential prognostic role of these molecules.

\section{Conflict of Interests}

The authors declare that they have no competing interests.

\section{References}

Abou Asa S, Mori T, Maruo K, Khater A, El-Sawak A et al. (2013). Analysis of genomic mutation and immunohistochemistry of platelet-derived growth factor receptors in canine vascular tumours. Veterinary and Comparative Oncology, doi: 10.1111/vco.12035.

Alvarez RH, Kantarjian HM, Cortes JE (2006). Biology of platelet-derived growth factor and its involvement in disease. Mayo Clinic Proceedings, 81, 1241-57. 
Aresu L, Aricò A, Comazzi S, Gelain ME, Riondato F et al. (2012). VEGF and MMP-9: biomarkers for canine lymphoma. Veterinary and Comparative Oncology, 1-8, doi: 10.1111/j.1476-5829.2012.00328.x.

Aresu L, Martini V, Rossi F, Vignoli M, Sampaolo M et al. (2013). Canine indolent and aggressive lymphoma: clinical spectrum with histologic correlation. Veterinary and Comparative Oncology. doi: 10.1111/vco.12048.

Aricò A, Giantin M, Gelain ME, Riondato F, Comazzi S et al. (2013). The role of vascular endothelial growth factor and matrix metalloproteinases in canine lymphoma: in vivo and in vitro study. BMC Veterinary Research, 9, 94.

Ferrara N, Gerber HP, LeCouter J (2003). The biology of VEGF and its receptors. Nature Medicine, 9, 669-76.

Halper J (2010). Growth factors as active participants in carcinogenesis: a perspective. Veterinary Pathology, 47, 77-97.

Heldin CH, Westermark B (1999). Mechanism of action and in vivo role of platelet-derived growth factor. Physiological Reviews, 79, 1283-316.

Karabatsou CL, Hsu LF, Phyliky RL, Li CY (2005). Autocrine expression of platelet-derived growth factor B in B cell chronic lymphocytic leukemia. Acta Haematologica, 114, 133-40.

Karabatsou K, Pal P, Dodd S, Mat A, Haylock B et al. (2006). Expression of survivin, plateletderived growth factor A (PDGF-A) and PDGF receptor alpha in primary central nervous system lymphoma. Journal of Neuro-Oncology, 79, 171-9.

Kisseberth WC, Nadella MVP, Breen M, Thomas R, Duke SE et al. (2007). A novel canine lymphoma cell line: a translational and comparative model for lymphoma research. Leukemia Research, 31, 1709-20.

Labidi SI, Ménétrier-Caux C, Chabaud S, Chassagne C, Sebban C et al. (2010). Serum cytokines in follicular lymphoma. Correlation of TGF- $\beta$ and VEGF with survival. Annals of Hematology, 89, 25-33.

Livak KJ, Schmittgen TD (2001). Analysis of relative gene expression data using real-time quantitative PCR and the 2(-Delta Delta C(T)) Method. Methods, 25, 402-8.

Maniscalco L, Iussich S, Morello E, Martano M, Biolatti B et al. (2013). PDGFs and PDGFRs in canine osteosarcoma: new targets for innovative therapeutic strategies in comparative oncology. Veterinary Journal, 195, 41-7.

Marconato L, Gelain ME, Comazzi S (2013). The dog as a possible animal model for human nonHodgkin lymphoma: a review. Hematological Oncology, 31, 1-9.

Moehler TM, Ho AD, Goldschmidt H, Barlogie B (2003). Angiogenesis in hematologic malignancies. Critical Reviews in Oncology/Hematology, 45, 227-44. 
Pedersen LM, Klausen TW, Davidsen UH, Johnsen HE (2005). Early changes in serum IL-6 and VEGF levels predict clinical outcome following first-line therapy in aggressive non-Hodgkin's lymphoma. Annals of Hematology, 84, 510-6.

Piccaluga PP, Agostinelli C, Zinzani PL, Baccarani M, Dalla Favera R et al. (2005). Expression of platelet-derived growth factor receptor alpha in peripheral T-cell lymphoma not otherwise specified. The Lancet Oncology, 6, 440.

Piccaluga PP, Rossi M, Agostinelli C, Ricci F, Gazzola A et al. (2014). Platelet-derived growth factor alpha mediates the proliferation of peripheral T-cell lymphoma cells via an autocrine regulatory pathway. Leukemia, doi: 10.1038/leu.2014.50.

Ponce F, Marchal T, Magnol JP, Turinelli V, Ledieu D et al. (2010). A morphological study of 608 cases of canine malignant lymphoma in France with a focus on comparative similarities between canine and human lymphoma morphology. Veterinary Pathology, 47, 414-33.

Renné C, Willenbrock K, Küppers R, Hansmann ML, Bräuninger A (2005). Autocrine- and paracrine-activated receptor tyrosine kinases in classic Hodgkin lymphoma. Blood, 105, 40519.

Ria R, Cirulli T, Giannini T, Bambace S, Serio G et al. (2008). Serum levels of angiogenic cytokines decrease after radiotherapy in non-Hodgkin lymphomas. Clinical and Experimental Medicine, 8, 141-5.

Ruan J, Luo M, Wang C, Fan L, Yang SN et al. (2013). Imatinib disrupts lymphoma angiogenesis by targeting vascular pericytes. Blood, 121, 5192-202.

Rütgen BC, Hammer SE, Gerner W, Christian M, de Arespacochaga AG et al. (2010). Establishment and characterization of a novel canine B-cell line derived from a spontaneously occurring diffuse large cell lymphoma. Leukemia Research, 34, 932-8.

Valli VE, San Myint M, Barthel A, Bienzle D, Caswell J et al. (2011). Classification of canine malignant lymphomas according to the World Health Organization criteria. Veterinary Pathology, 48, 198-211. 


\section{Figure legend}

Fig. 1. PDGF-A, PDGF-B, PDGFR- $\alpha$ and PDGFR- $\beta$ mRNA expression in control lymph nodes, DLBCLs, PTCLs and T-LBLs. Data are expressed as the mean \pm standard deviation

a, b, c Significant differences between control lymph nodes and DLBCLs, control lymph nodes and PTCLs \& T-LBLs, PTCLs \& T-LBLs and DLBCLs, respectively (Kruskal-Wallis test followed by Dunn's post-test, $p<0.05)$.

Fig. 2. PDGF-A, PDGF-B, PDGFR- $\alpha$ and PDGFR- $\beta$ protein expression in control lymph nodes, DLBCLs, PTCLs and T-LBLs. Data are expressed as the mean \pm standard deviation a, b, c Significant differences between DLBCLs and control lymph nodes, PTCLs \& T-LBLs and control lymph nodes, PTCLs \& T-LBLs and DLBCLs, respectively (Kruskal-Wallis test followed by Dunn's post-test, $p<0.05)$.

Fig. 3. Representative control lymph node, DLBCL and PTCL show cytoplasmic staining in lymphocytes for PDGF-A (a, b, c, respectively), for PDGF-B (d, e, f, respectively), PDGFR- $\alpha$ (g, h, $\mathrm{i}$, respectively) and PDGFR- $\beta$ (1, j, k, respectively). Original magnification, X200, Inset (d, g and j), X400. 
Table 1

Primer sequences used for $q R T$-PCR amplification

\begin{tabular}{|c|c|l|}
\hline Genes & Accession Number & \multicolumn{1}{c|}{ Primer Sequence (5'-3') } \\
\hline PDGF-A & NM_001190172.1 & $\begin{array}{l}\text { F: TTTGGAAGCAAGTCTGAGAGCC } \\
\text { R: TGGCCTCCTCAATGCTTCTT }\end{array}$ \\
\hline PDGF-B & NM_001003383.1 & $\begin{array}{l}\text { F: CCGAGTTGGACCTGAATTTG } \\
\text { R: GTCTTGCACTCAGCGATCAT }\end{array}$ \\
\hline PDGFR- $\boldsymbol{\alpha}$ & AY525124.2 & $\begin{array}{l}\text { F: TTTCCCTTGGCGGCACAC } \\
\text { R: GTCAGGCTTGGCCATCCG }\end{array}$ \\
\hline PDGFR- $\boldsymbol{\beta}$ & NM_001003382.1 & $\begin{array}{l}\text { F: CACGCCTCTGACGAGATTTATG } \\
\text { R: CTCGAGAAGCAGCACCAGCT }\end{array}$ \\
\hline
\end{tabular}


Table 1 Supplementary Material

PDGF-A, PDGF-B, PDGFR- $\alpha$ and PDGFR- $\beta$ mRNA expression in control lymph nodes, reactive hyperplasia, DLBCLs, PTCLs, T-LBLs and cell lines.

\begin{tabular}{|c|c|c|c|c|}
\hline & PDGF-A & PDGF-B & PDGFR- $\alpha$ & PDGFR- $\beta$ \\
\hline B-cell lymphoma (DLBCL) $(n=19)$ & $0.83 \pm 0.41$ & $0.56 \pm 0.36$ & $0.75 \pm 0.46$ & $0.44 \pm 0.26$ \\
\hline T-cell lymphoma $(n=8)$ & $0.79 \pm 0.48$ & $2.26 \pm 1.96$ & $0.67 \pm 0.36$ & $0.68 \pm 0.35$ \\
\hline PTCL $(n=5)$ & $0.69 \pm 0.34$ & $2.13 \pm 2.25$ & $0.66 \pm 0.48$ & $0.52 \pm 0.38$ \\
\hline T-LBL $(n=3)$ & $0.89 \pm 0.48$ & $2.37 \pm 1.63$ & $0.69 \pm 0.13$ & $0.82 \pm 0.14$ \\
\hline reactive hyperplasia $\mathbf{T 0}^{*}(\mathrm{n}=9)$ & $1.95 \pm 1.13$ & $2.01 \pm 1.36$ & $2.29 \pm 1.54$ & $1.55 \pm 0.85$ \\
\hline control lymph nodes $(\mathrm{n}=5)$ & $1.93 \pm 0.73$ & $0.91 \pm 0.51$ & $3.52 \pm 0.71$ & $1.35 \pm 0.50$ \\
\hline reactive hyperplasia $\mathbf{T} 1 * *(\mathrm{n}=8)$ & $2.05 \pm 1.01$ & $1.81 \pm 1.04$ & $2.89 \pm 1.92$ & $1.90 \pm 0.94$ \\
\hline CLBL-1 & n.q. & n.q. & 0 & n.q. \\
\hline OSW & $2.22 \pm 0.15$ & 0 & $3.46 \pm 0.22$ & 0 \\
\hline
\end{tabular}

Data are expressed as the mean \pm standard deviation

n.q. = not quantifiable

* At the time of admission

** At the end of treatment 
Table 2 Supplementary Material

PDGF-A, PDGF-B, PDGFR- $\alpha$ and PDGFR- $\beta$ protein expression in control lymph nodes, reactive hyperplasia, DLBCLs, PTCLs and T-LBLs.

\begin{tabular}{|ccccc|}
\hline & PDGF-A & PDGF-B & PDGFR- $\alpha$ & PDGFR- $\beta$ \\
\hline B-cell lymphoma (DLBCL) $(\mathrm{n}=19)$ & $125.8 \pm 50.6$ & $15.9 \pm 34.1$ & $291.5 \pm 21.9$ & $289.8 \pm 19.8$ \\
T-cell lymphoma (n=8) & $77.1 \pm 40.4$ & $213.2 \pm 43.2$ & $277.4 \pm 28$ & $271.2 \pm 29.2$ \\
PTCL (n=5) & $90.2 \pm 41.2$ & $214.5 \pm 57.7$ & $284.4 \pm 18.2$ & $274.6 \pm 27$ \\
T-LBL (n=3) & $66.1 \pm 28.3$ & $212 \pm 27.6$ & $271.6 \pm 17.3$ & $268.3 \pm 5.8$ \\
\hline reactive hyperplasia T0* $(\mathrm{n}=9)$ & $79 \pm 33.1$ & $10.2 \pm 5.2$ & $150.9 \pm 40.3$ & $145 \pm 65.9$ \\
\hline control lymph nodes $(\mathrm{n}=5)$ & $82.1 \pm 46.6$ & $11.3 \pm 22$ & $189.9 \pm 65.9$ & $166.4 \pm 82.7$ \\
\hline reactive hyperplasia T1** $(\mathrm{n}=8)$ & $59.1 \pm 20.3$ & $6.4 \pm 12.3$ & $138.5 \pm 58$ & $188.4 \pm 72.6$ \\
\hline
\end{tabular}

Data are expressed as the mean \pm standard deviation

* At the time of admission

** At the end of treatment 